\title{
The in-situ Laser Scattering Electrospray Ionization Mass Spectrometry and Its Application in the \\ Mechanism Study of Photo-Induced Direct C-H Arylation of Heteroarenes
}

\author{
Wanpeng Ai, ${ }^{a}$ Qirong Yang, a Yunpeng Gao, ${ }^{a}$ Xiaoyun Liu, ${ }^{b}$ Huwei Liu, a and Yu Bai ${ }^{*}$ a \\ a. Beijing National Laboratory for Molecular Sciences, Key Laboratory of Bioorganic Chemistry and \\ Molecular Engineering of Ministry of Education, Institute of Analytical Chemistry, College of Chemistry \\ and Molecular Engineering, Peking University, Beijing 100871, P. R. China \\ b. Department of Microbiology, School of Basic Medical Sciences, Peking University Health Science \\ Center, Beijing 100191, China \\ *Corresponding author: Dr. Yu Bai, E-mail: yu.bai@pku.edu.cn
}

\section{Table of content}

Synthesis of the substrates benzenediazonium tetrafluoroborate..............................S2

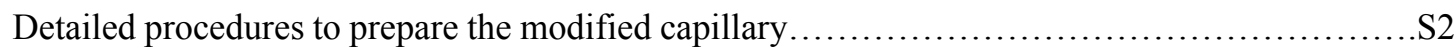

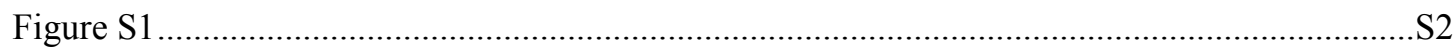

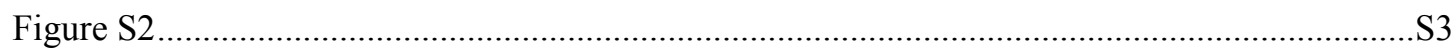

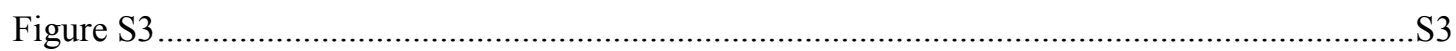

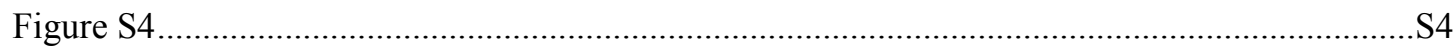

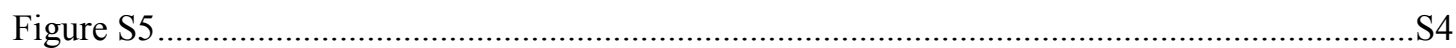

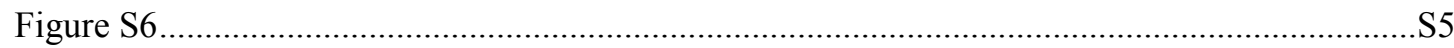




\section{Synthesis of the substrates benzenediazonium tetrafluoroborate}

The aniline $(0.01$ moles) was dissolved in a mixture of $40 \%$ fluoroboric acid $(3.76 \mathrm{~mL})$ and distilled water $(2.24 \mathrm{~mL})$. After cooling to $0{ }^{\circ} \mathrm{C}$, sodium nitrite $(0.69 \mathrm{~g})$ in $3 \mathrm{~mL}$ of distilled water was added in dropwise. The mixture was stirred for 30 minutes and the thick precipitate was collected and re-dissolved in acetone. The benzenediazonium tetrafluoroborate was then precipitated by the addition of diethyl ether.

\section{Detailed procedures to prepare the modified capillary}

After removal of the polyimide coating of the $\sim 9 \mathrm{~cm}$ capillary (Figure S1a), its tip was pulled using a P-2000 laser pipette puller (Sutter Instrument Co., Novato, CA, USA). Then it was immersed for about one minute in the GO water suspension contained in a cap of $200 \mu \mathrm{L}$ test tube with two notches (Figure S1b). The prepared capillary and the enlarged part was shown in Figure S1c (Figure S1d). Figure S1d showed the capillary after the laser-irradiation and its enlarged part.

(a)

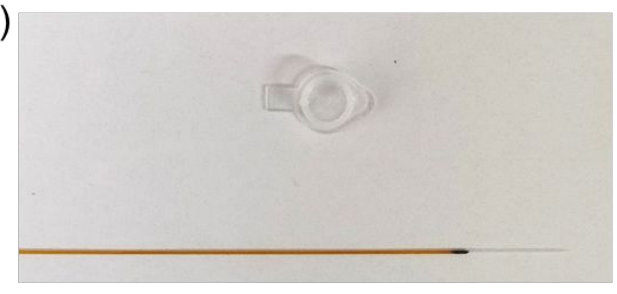

(b)

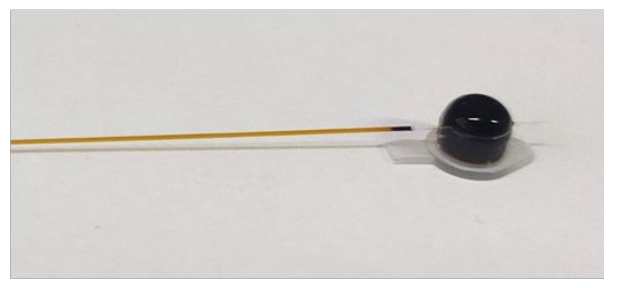

(c)

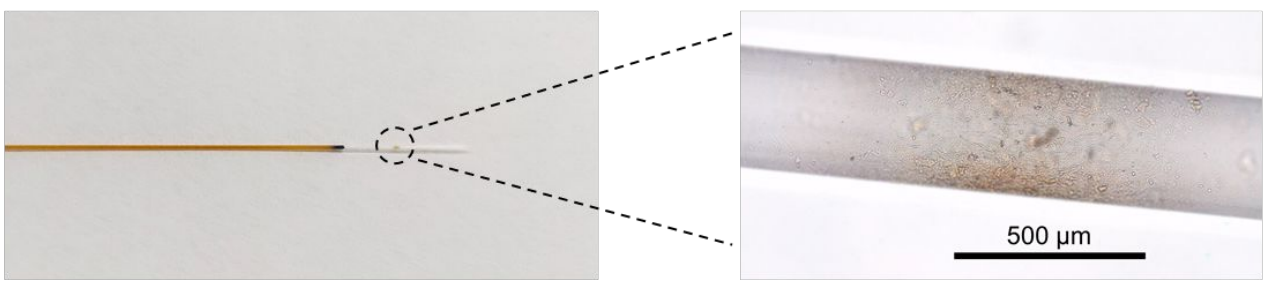

(d)

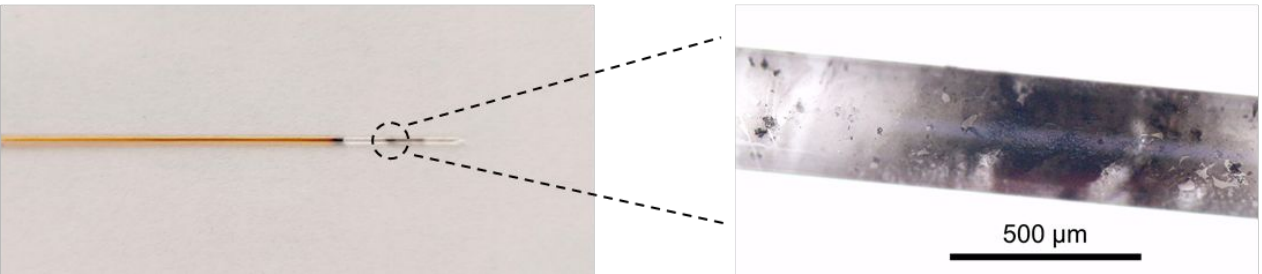

Figure S1. (a) The photo of the capillary and the cap of $200 \mu \mathrm{L}$ test tube; (b) The immersion of the capillary in GO suspension; (c) Prepared capillary and the enlarged part that coated with GO film; (e) Laser-irradiated capillary and the enlarged part that coated with GO film. 


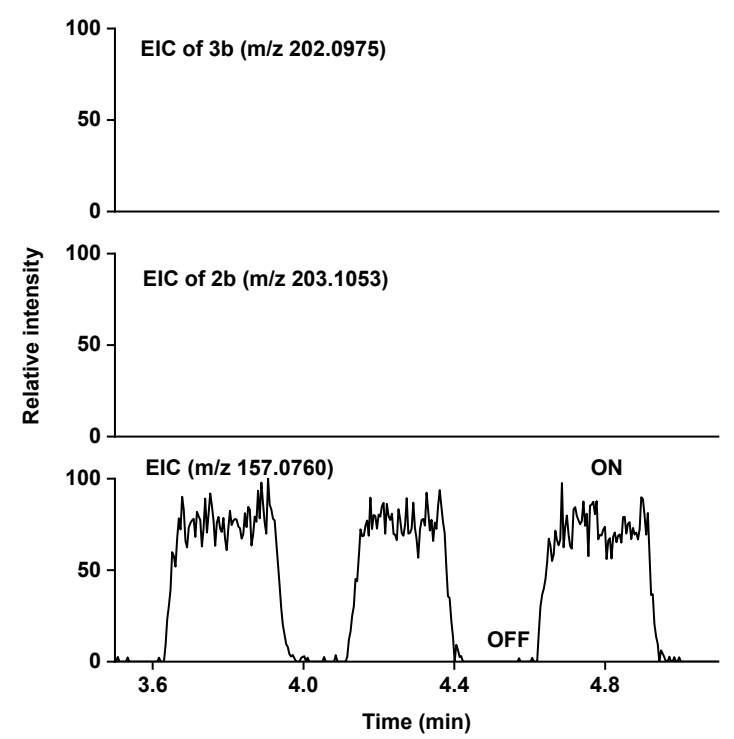

Figure S2. EICs of the intermediates at $\mathrm{m} / \mathrm{z} 202.0975,203.1053$ and 157.0760 with direct laser irradiation MS setup.
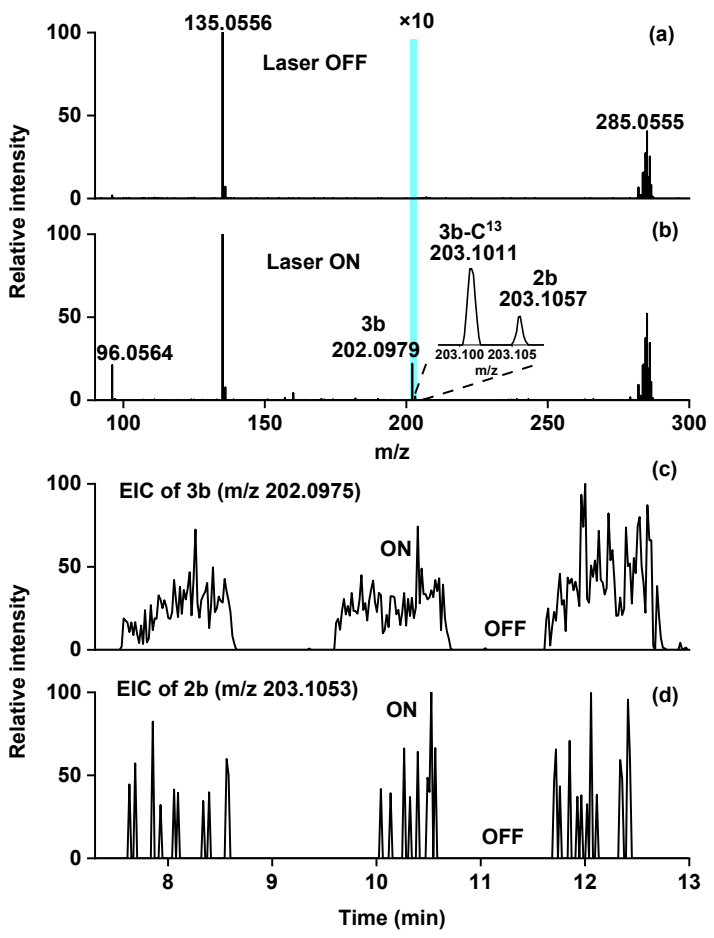

Figure S3. MS spectra of 4-methoxybenzenediazonium tetrafluoroborate (24 ppm), pyrazin-2amine (3.6 ppm) and $\left[\mathrm{Ru}(\mathrm{II})(\mathrm{bpy})_{3}\right] \mathrm{Cl}_{2}(8 \mathrm{ppm})$ in $\mathrm{CH}_{3} \mathrm{OH}$ with the (a) laser off mode and (b) laser on mode (the insert: difference between of $\mathbf{3} \mathbf{b}-\mathrm{C}^{13}$ and $\left.\mathbf{2 b}\right)$; EICs of the (c) $\mathbf{3 b}(\mathrm{m} / \mathrm{z} 202.0975)$ and (d) $\mathbf{2 b}(\mathrm{m} / \mathrm{z} 203.1053)$ with laser irradiation 

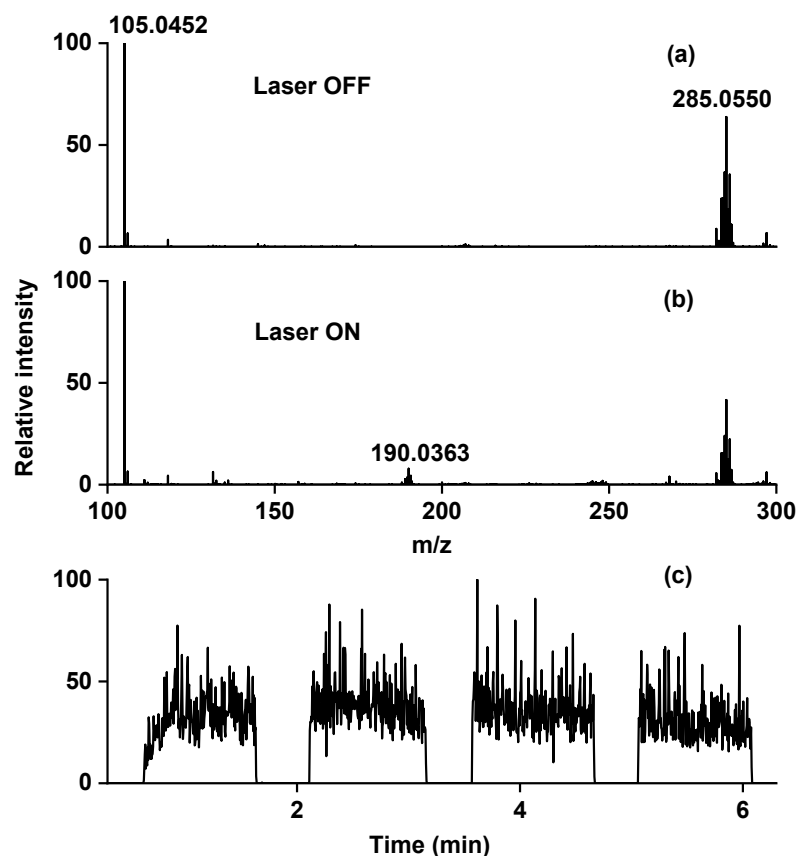

Figure S4. MS spectra of benzenediazonium tetrafluoroborate $(30 \mathrm{ppm})$ and $\left[\mathrm{Ru}(\mathrm{II})(\mathrm{bpy})_{3}\right] \mathrm{Cl}_{2}(8$ ppm) in $\mathrm{CH}_{3} \mathrm{CN}$ with (a) the laser off mode and (b) the laser on mode; (c) EIC of the intermediate $\mathrm{Ru}(\mathrm{bpy}) 3_{3}^{3+}$ with laser irradiation

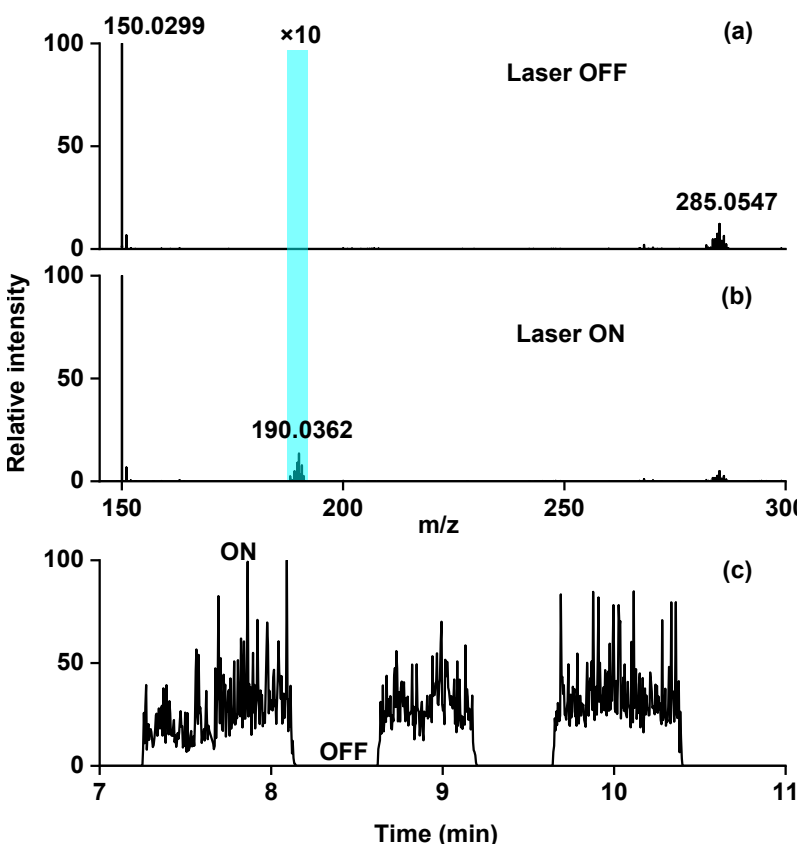

Figure S5. MS spectra of 4-nitrobenzenediazonium tetrafluoroborate $(15 \mathrm{ppm})$ and $\left[\mathrm{Ru}(\mathrm{II})(\mathrm{bpy})_{3}\right] \mathrm{Cl}_{2}(2 \mathrm{ppm})$ in $\mathrm{CH}_{3} \mathrm{CN}$ with (a) the laser off mode and (b) the laser on mode; (c) EIC of the intermediate $\mathrm{Ru}(\mathrm{bpy})_{3}{ }^{3+}$ with laser irradiation. 


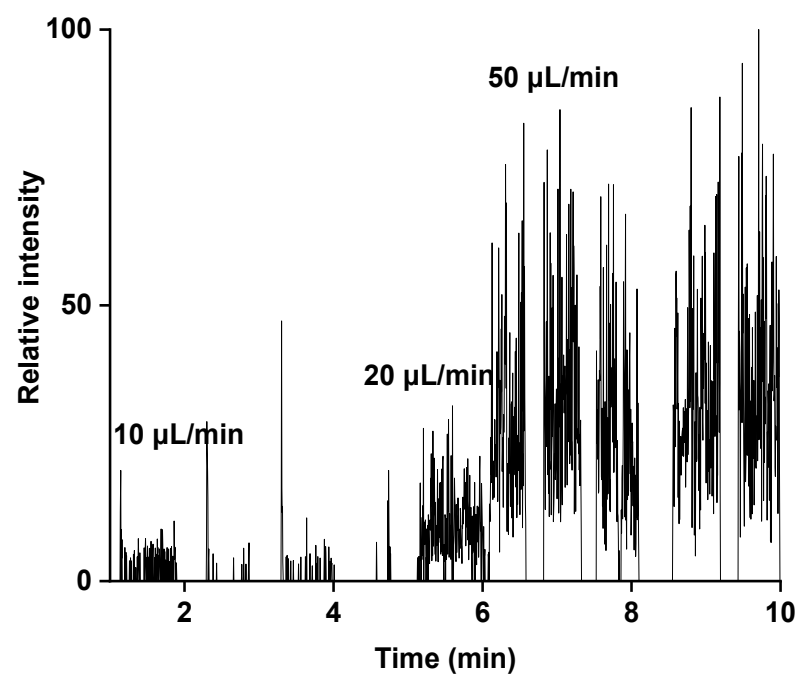

Figure S6. EIC of the intermediates of $\mathrm{Ru}(\mathrm{bpy})_{3^{3+}}$ in the methanol solution of 4nitrobenzenediazonium tetrafluoroborate $(100 \mathrm{ppm})$ and $\left[\mathrm{Ru}(\mathrm{II})(\mathrm{bpy})_{3}\right] \mathrm{Cl}_{2}(13 \mathrm{ppm})$ with laser irradiation with different flow rate. 